\title{
ANÁLISE DA VARIABILIDADE DIÁRIA DA PRECIPITAÇÃO EM ÁREA DE PASTAGEM PARA A ÉPOCA CHUVOSA DE 1999 - PROJETO TRMM/LBA
}

\author{
Julio TOTA ${ }^{1}$, Gilberto FISCH ${ }^{2}$, José FUENTES ${ }^{3}$, Paulo Jorge de OLIVEIRA ${ }^{1}$ \\ Michael GARSTANG ${ }^{3}$, Ryan HEITZ ${ }^{3}$, Jeffrey SIGLER ${ }^{3}$
}

\begin{abstract}
RESUMO - Como parte das investigações do experimento TRMM/LBA na Amazônia, medidas de chuva foram obtidas continuamente sobre uma área de pastagem. Neste trabalho foram analisados dados de precipitação durante o período compreendido entre 10 de janeiro a 28 de fevereiro de 1999 (periodo chuvoso). Os totais mensais de precipitação para o período estudado foram de 322,3 e 468,6 mm para janeiro e fevereiro, respectivamente. As análises da distribuição de frequência para os eventos inferiores à $1 \mathrm{~mm} / \mathrm{h}$ e superiores à $1,3,5,10,15 \mathrm{e}$ $20 \mathrm{~mm} \cdot \mathrm{h}^{-1}$, mostraram diferentes distribuição temporal. Os eventos inferiores à $1 \mathrm{~mm} \cdot \mathrm{h}^{-1}$ apresentaram uma distribuição temporal mais regular, porém representando apenas cerca de $3 \%$ do volume total de precipitação $(27 \mathrm{~mm})$. Os eventos com precipitação superiores à $1,3,5$ $\mathrm{mm} \cdot \mathrm{h}^{-1}$ apresentaram um padrão similar entre $\mathrm{si}$, sendo que os casos mais freqüentes ocorreram durante a madrugada e final da tarde. Esses eventos representaram cerca de 97,91 e $86 \%$ do total da precipitação, respectivamente. Finalmente, os eventos acima de 10,15 e $20 \mathrm{~mm} \cdot \mathrm{h}^{-1}$ totalizaram, respectivamente, 73,68 e $62 \%$ do total da precipitação, e as maiores frequências relativas concentraram-se entre 2 às $4 \mathrm{HL}$ e 15 às $17 \mathrm{HL}$. A precipitação observada pode ser classificada em 2 sistemas distintos de produção de chuva: convecção isolada local e sistemas convectivos de mesoescala, os quais provocaram máximos de precipitação às $16 \mathrm{e} 4 \mathrm{HL}$, respectivamente.
\end{abstract}

Palavras-Chave: Chuva, Amazônia, Ciclo diário

The Analysis of the Daily Rainfall in a Pasture Site During the Wet Season of 1999 - TRMM/ LBA Project

ABSTRACT - As part of the investigations of the experiment TRMM/LBA in Amazônia, measurements of the rain were obtained in a pasture site. In this work precipitation distribution were analyzed during the period from January 10 to February 28,1999 (wet season). The monthly totals of precipitation for the period were 322.3 and $468.6 \mathrm{~mm}$ for January and February, respectively. The analyses of the frequency distribution for the events lower than $1 \mathrm{~mm} . \mathrm{h}$ ${ }^{1}$ and higher than $1,3,5,10,15$ and $20 \mathrm{~mm} \cdot \mathrm{h}^{-1}$ showed different time distribution. The events lower than $1 \mathrm{~mm} \cdot \mathrm{h}^{-1}$ presented a more regular temporary distribution, even representing only $3 \%$ of the total volume of precipitation $(27 \mathrm{~mm})$. The events with precipitation above $1,3,5$ $\mathrm{mm} . \mathrm{h}^{-1}$ presented a similar pattern among them, and the most frequent cases ocurred during late afternoon. Those events represented about 97,91 and $86 \%$ of the total of the precipitation, respectively. Finally, the events above 10,15 and $20 \mathrm{~mm} \cdot \mathrm{h}^{-1}$ represented 73,68 and $62 \%$ of the total of the precipitation respectively, and the largest relative frequencies concentrated among 2 up to $4 \mathrm{HL}$ and 15 up to $17 \mathrm{HL}$. The rainfall observed can be classified in 2 distint system: local convection and mesoscale convective system, which provoke peaks at $16 \mathrm{LT}$ and $4 \mathrm{LT}$, respectively.

Key-words: Rain, Amazonia, Daily cycle

${ }^{1}$ Centro de Previsão e Estudos Climáticos (CPTEC/INPE)-Cachoeira Paulista, 12630-000, SP, Brasil. E-mail: tota@met.inpe.br

${ }^{2}$ Centro Técnico Aeroespacial (CTA/IAE-ACA), São José dos Campos, 12228-904, SP, Brasil.

E-mail: gfisch@iae.cta.br

${ }^{3}$ University of Virginia (UVA), Charlottesville, Virginia, USA. E-mail: jf6s@virginia.edu. 


\section{INTRODUÇÃO}

A precipitação é uma das variáveis mais importantes no estudo da caracterização do clima de uma região. Além de seu papel no ciclo hidrológico, esta variável também influencia na manutenção do equilibrio do meio ambiente e biodiversidade. A Amazônia possui um papel importante na determinação do clima global, pela sua vasta bacia hidrográfica situada na região equatorial e também por ser uma fonte de energia aos processos atmosféricos da circulação atmosférica de grande escala (Molion, 1987).

Atualmente há um grande interesse científico na avaliação das alterações que o desmatamento da floresta tropical Amazônica possa provocar nos climas regional e global. Alguns estudos de simulação numérica do clima usando Modelos de Circulação Geral da Atmosfera (MCGAs) foram realizados, os quais foram calibrados com dados obtidos em experimentos de campo na região (por exemplo o Projeto ABRACOS veja Gash e Nobre, 1997). Os resultados desses estudos sugerem que a troca de vegetação provoca um aumento da temperatura do ar (variando de 0,6 a $2,0{ }^{\circ} \mathrm{C}$ ), uma redução nos totais de evaporação e de precipitação (de 20 a $30 \%$ ) e que a estação seca na Amazônia seria mais prolongada após o desmatamento (Nobre et al., 1991; Fisch et al., 1997).
Uma revisão do clima e das principais interações dos fenômenos meteorológicos em várias escalas sobre a região Amazônica pode ser encontrado em Fisch et al. (1998).

Devido a forte interação florestaatmosfera, existe uma alta variabilidade da distribuição de chuva ao longo do dia na região Amazônica. Entretanto, grande parte desta área de floresta natural tem sido convertida em área de pastagem (fazenda), cuja distribuição e variabilidade diária das chuvas é ainda desconhecida e pouco abordada pelos estudos de simulação climática citados anteriormente. Estudos observacionais no contexto da variabilidade horária de precipitação em áreas desmatadas são poucos, tais como Dias de Paiva (1996) e Ferreira da Costa et al. (1998).

O Projeto TRMM-LBA foi um experimento de campo, inserido no Projeto LBA (Experimento de Grande Escala na Biosfera-Atmosfera na Amazônia) - INPE, que coletou dados de superfície e de ar superior visando a calibração de sensores do satélite NASA-TRMM (Tropical Rain Measuring Mission). Dentre os dados desse experimento, realizado no Estado de Rondônia durante a estação úmida, foram medidas as taxas de precipitação em uma área de pastagem. O objetivo deste estudo é o de caracterizar a distribuição da precipitação (total mensal, ciclo diário, sistemas produtores de precipitação, 
etc.) durante a estação chuvosa, utilizando os dados coletados na campanha de campo do Projeto TRMM/LBA (jan-fev/1999).

\section{MATERIAL E MÉTODOS}

A coleta de dados foi realizada em uma área de pastagem, (Fazenda Nossa Senhora de Aparecida) localizada em $10^{\circ} 45^{\prime}$ S e $62^{\circ} 22^{\prime} \mathrm{W}$, 293 metros acima do nível do mar, no municipio de Ouro Preto D'Oeste, a $50 \mathrm{~km}$ de Ji-Paraná e com cobertura vegetal do tipo graminea (Brachiaria brizantha). Esta área possui uma orografia com elevações de cerca de 150 metros (Fig. 1).

Para caracterizar a climatologia da região de Ji-Paraná, na Figura 2 são mostradas as médias mensais de precipitação para o período de 1982 a
1996, medidos a partir de uma estação convencional localizada em Ouro Preto D'Oeste, cerca de $50 \mathrm{~km}$ do experimento (Ferreira da Costa et al., 1998). Uma forte sazonalidade pode ser observada na distribuição da precipitação, com o período mais chuvoso (novembro-abril) apresentando valores médios mensais acima de $200 \mathrm{~mm}$ mês $^{-1}$. Durante a estação seca (junho-agosto), o total mensal médio raramente alcança valores de $20 \mathrm{~mm}^{\text {mês }}{ }^{-1}$.

Foram utilizados dados de totais de precipitação coletados, a cada minuto, através de um pluviômetro de báscula (Tipping Bucket Rain Gauge), de 10 de janeiro a 28 de fevereiro de 1999 (estação chuvosa). Os dados medidos a cada minuto foram integrados para cada hora do dia. A partir deste conjunto de dados foi

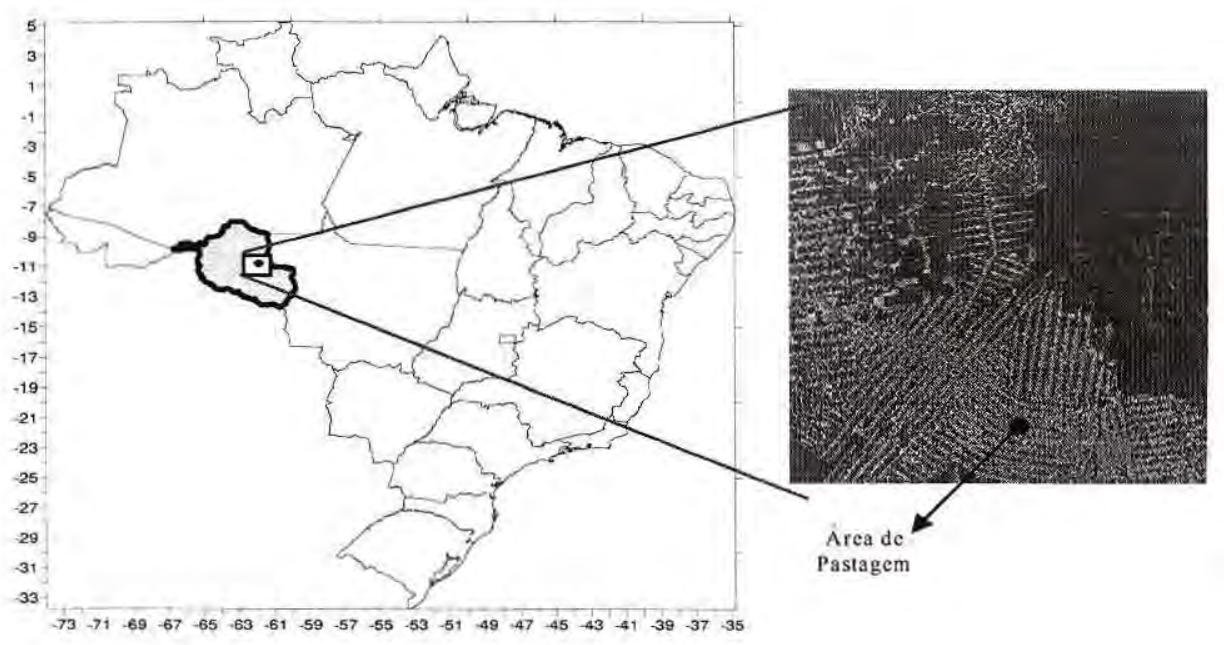

Figura 1. Localização geográfica do sítio experimental no Estado de Rondônia. Fonte: Adaptado de Tóta (1998). 


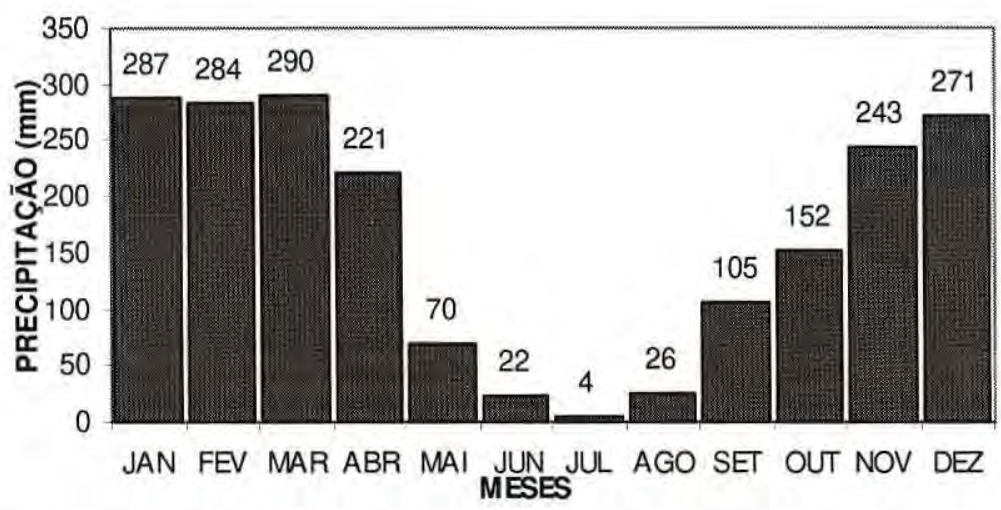

Figura 2. Climatologia da Precipitação para Ouro Preto D'Oeste (RO), período 1982-1996.

Fonte: Ferreira da Costa et al. (1998)

calculada a frequência de ocorrência da precipitação para casos com taxas inferior e superior a $1 \mathrm{~mm} / \mathrm{h}$ superiores à $3,5,10,15$, e $20 \mathrm{~mm} / \mathrm{h}$. Dessa forma, foram definidos o número de eventos de precipitação que ocorreram em cada hora para os dias estudados. Para definir a frequência relativa de precipitação, foi utilizada a relação entre o número de eventos de precipitação de cada hora e o total de eventos nas 24 horas, dada por:

$$
\mathrm{FR}=\frac{\mathrm{F}_{\mathrm{h}}}{\mathrm{F}_{24}} \times 100
$$

onde, FR é a frequência relativa da precipitação, $\mathrm{F}_{\mathrm{h}}$ é o número de eventos ocorridos numa dada hora, e $\mathrm{F}_{24}$ é o número de eventos ocorridos durante as 24 horas do dia. Além disso, também foram feitos cálculos da frequência relativa para intervalos de 6 horas, divididas como periodos entre 1-6, 7-12, 13-18, 19-24 hora local (HL).

\section{RESULTADOS}

A precipitação total para os períodos estudados (10-31 de janeiro e 1-28 de fevereiro de 1999) foram de 322,3 e de $468,6 \mathrm{~mm}$ (total de 790,9 $\mathrm{mm}$ ), respectivamente. Esses totais mensais caracterizam bem a estação chuvosa da região e foram superiores aos da normal climatológica (Fig. 2). Em relação aos totais diários de precipitação (Fig. 3-b), observou-se que o dia $25 / 02$ foi o mais chuvoso com um total de $112,5 \mathrm{~mm}$, seguido pelo dia 20/01 com um total diário de $81,8 \mathrm{~mm}$. A série temporal dos totais horários de precipitação (Fig. 3-a) mostram também que as chuvas concentraram-se às $16 \mathrm{HL}$ (tarde) para o dia $20 / 01$, enquanto que para $25 / 02$ foram predominantes às $19 \mathrm{HL}$ (noite). Além disso, na Figura 3-a, pode ser visto que durante o mês de fevereiro houveram eventos com forte precipitação durante a madrugada (dias 15 e 18/02).

Vale ressaltar que o total de precipitação entre 10 e $20 \mathrm{HL}$ é cerca 
de $66,6 \%(526,8 \mathrm{~mm})$ do total acumulado durante o experimento, enquanto que o período entre 21 e 09 HL correspondeu a $33,4 \%(264,1$ $\mathrm{mm})$. Essa é uma importante informação da estação chuvosa durante o TRMM-LBA, uma vez que a chuva durante a madrugada ocorreu devido a formação de Sistemas Convectivos de Mesoescala (SCM) (15 e 18/02), enquanto que as chuvas de formação local (convectiva) predominaram durante a maior parte do período analisado, principalmente entre $20 / 01$ e $8 / 02$. O período entre 8 e 14/02 apresentou-se como uma pequena estiagem, com pouquíssima precipitação $(10 \mathrm{~mm})$. A chuva convectiva local caracteriza-se por uma convecção profunda porém isolada, produzindo chuvas intensas mas de curta duração (1-2 horas, no máximo). Os SCM's formam-se da organização da convecção local com características estratiformes, cobrindo ampla área espacial e apresentando chuvas fracas a moderadas. Estes sistemas não produzem nuvens cumuliformes de grande desenvolvimento vertical como aqueles tipos característicos da

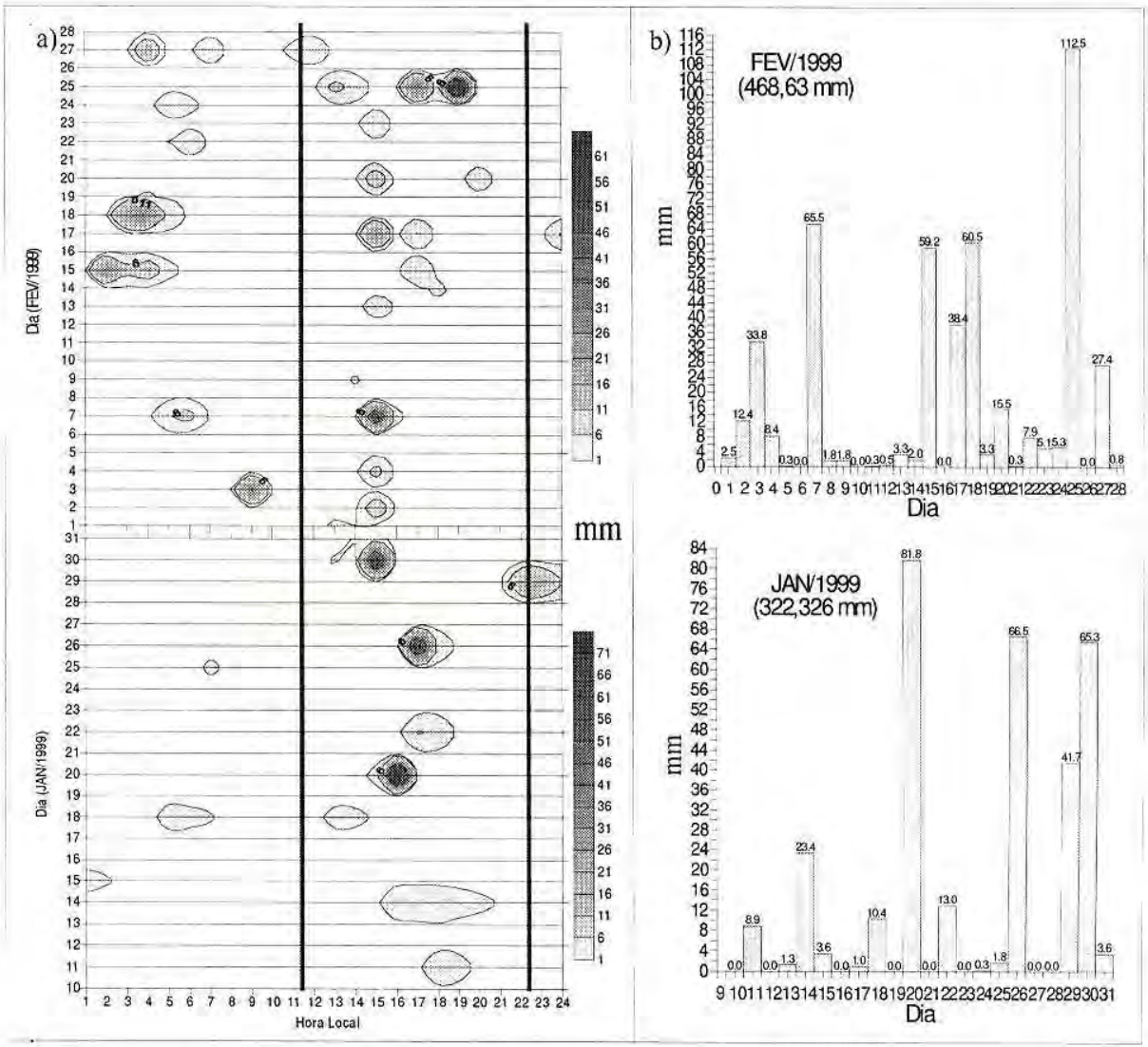

Figura 3. Distribuição horária (a) e diária (b) de precipitação de 10 janeiro a 28 de fevereiro 1999. 
convecção local.

As análises da distribuição de frequência da precipitação foram feitas para os eventos onde a ocorrência de precipitação foi inferior a $1 \mathrm{~mm} \cdot \mathrm{h}^{-1} \mathrm{e}$ superior a $1,3,5,10,15$ e $20 \mathrm{~mm} \cdot \mathrm{h}^{-1}$. Na Figura 4, pode-se observar que para eventos inferiores a $1 \mathrm{~mm} / \mathrm{h}$ (71 casos), a distribuição foi regular cobrindo praticamente todos os horários do dia. Nesta Figura é possivel visualizar uma leve predominância da ocorrência durante a madrugada (principalmente durante o mês de fevereiro), talvez relacionada a atuação de fenômenos de meso e/ou larga escala (particularmente os dias 15 e 18/02). O restante da precipitação concentrouse durante o período de máxima convecção do ciclo diurno, o qual é característico da região. Entretanto, em termos de volume, os casos com taxa de chuva inferior a $1 \mathrm{~mm} \cdot \mathrm{h}^{-1}$ representam apenas $3,4 \%$ do total de precipitação do período analisado (27,2 mm).

Para os eventos com taxas de precipitação superiores a 1,3 e 5

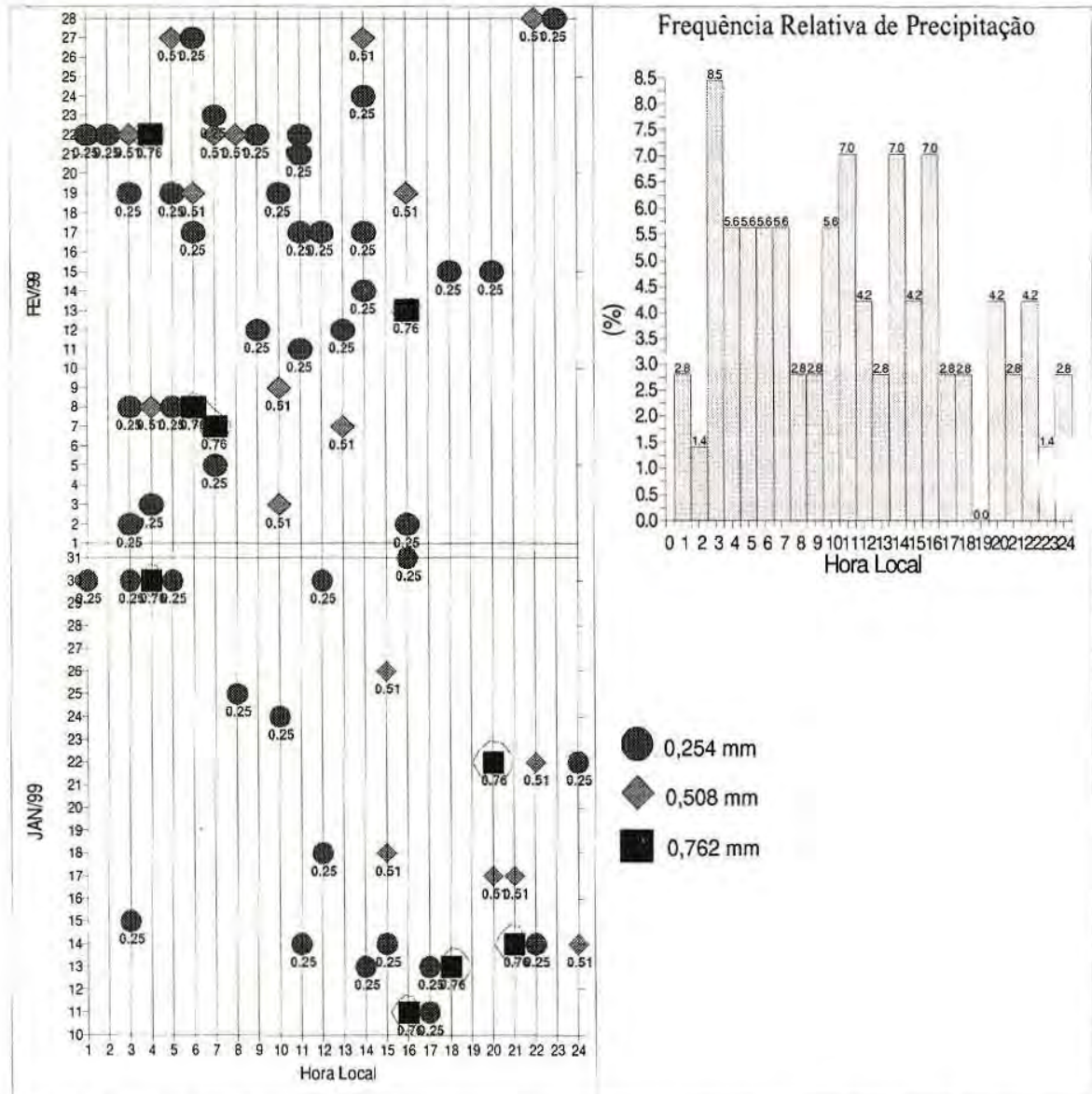

Figura 4. Série Temporal e Frequência Relativa da Precipitação inferior à $1 \mathrm{~mm} \cdot \mathrm{h}^{-1}$. 
$\mathrm{mm} \cdot \mathrm{h}^{-1}$ (com 71, 43, e 34 casos), a frequência relativa de precipitação foi concentrada em dois máximos (Distribuição Bimodal), sendo um máximo secundário durante a madrugada e um outro máximo principal à tarde, conforme mostram as Figuras 5-a e 6-a. Esses resultados mostram uma frequência relativa de precipitação consistente com o estudo feito por Ferreira da Costa et al. (1998), entretanto com magnitude superiores. Em termos de volume de precipitação, estes casos representaram cerca de 97,91 e $86 \%$ do total da precipitação, ou seja, 764, 717, $684 \mathrm{~mm}$.

Conforme pode ser visto,

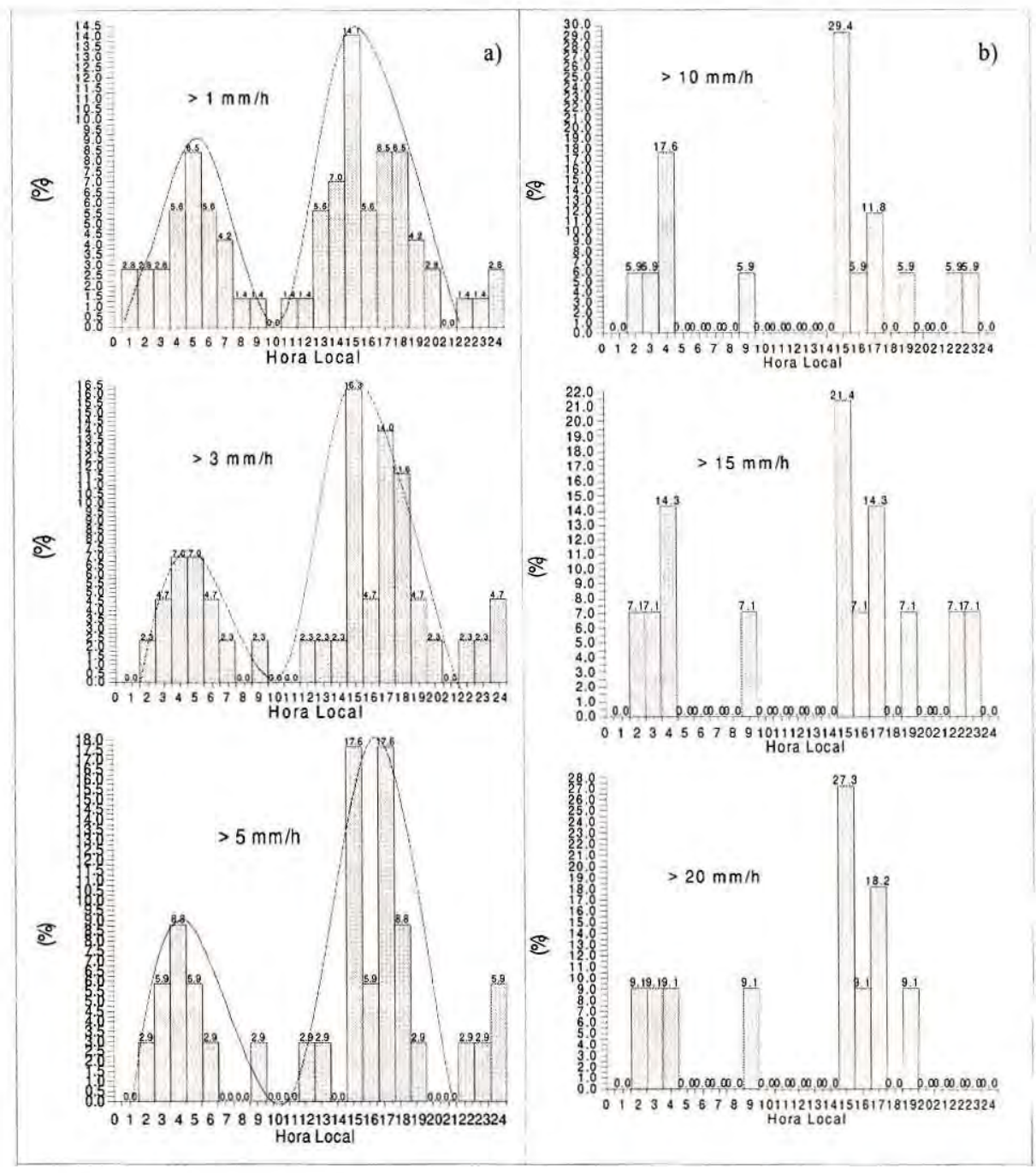

Figura 5. Frequência relativa de precipitação. 
respectivamente, para os eventos acima de 10,15 e $20 \mathrm{~mm} \cdot \mathrm{h}^{-1}(17,14$, e 11 casos), a frequência relativa de precipitação foi ainda mais concentrada em dois máximos às $4 \mathrm{e}$ $15 \mathrm{HL}$ (Figuras 5-b e 6-b). Na série temporal dessas taxas de precipitação, mostrada na Figura 6-b, fica evidente a concentração e intensidade da precipitação durante o período de convecção diurna local. Estes eventos representam, apesar do reduzido número de casos, cerca de 73,68 e $62 \%$ do total da precipitação, ou seja, 577,541 e $489 \mathrm{~mm}$. Durante a madrugada de 15 e 18/02 pode-se também observar nestas Figuras eventos com chuvas intensas que, como mencionado anteriormente, podem estar associados à atuação de sistemas de meso ou larga escala sobre a região.

A Figura 7 mostra a frequência relativa integrada em intervalos de 6
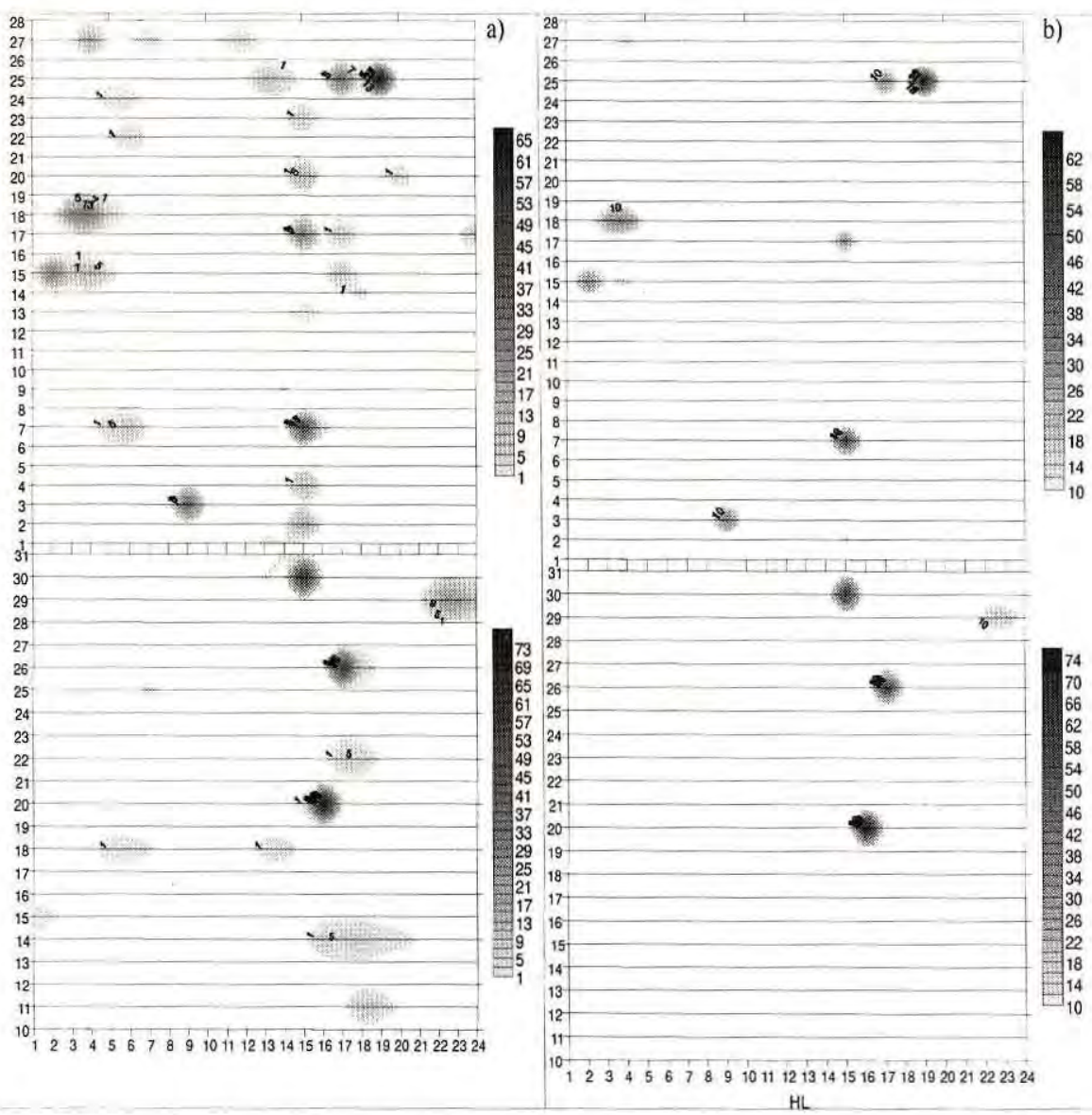

Figura 6. Série temporal e frequência relativa de precipitação superior à $1 \mathrm{~mm} \cdot \mathrm{h}^{-1}$ (a) e $10 \mathrm{~mm} \cdot \mathrm{h}^{-1}(\mathrm{~b})$. 


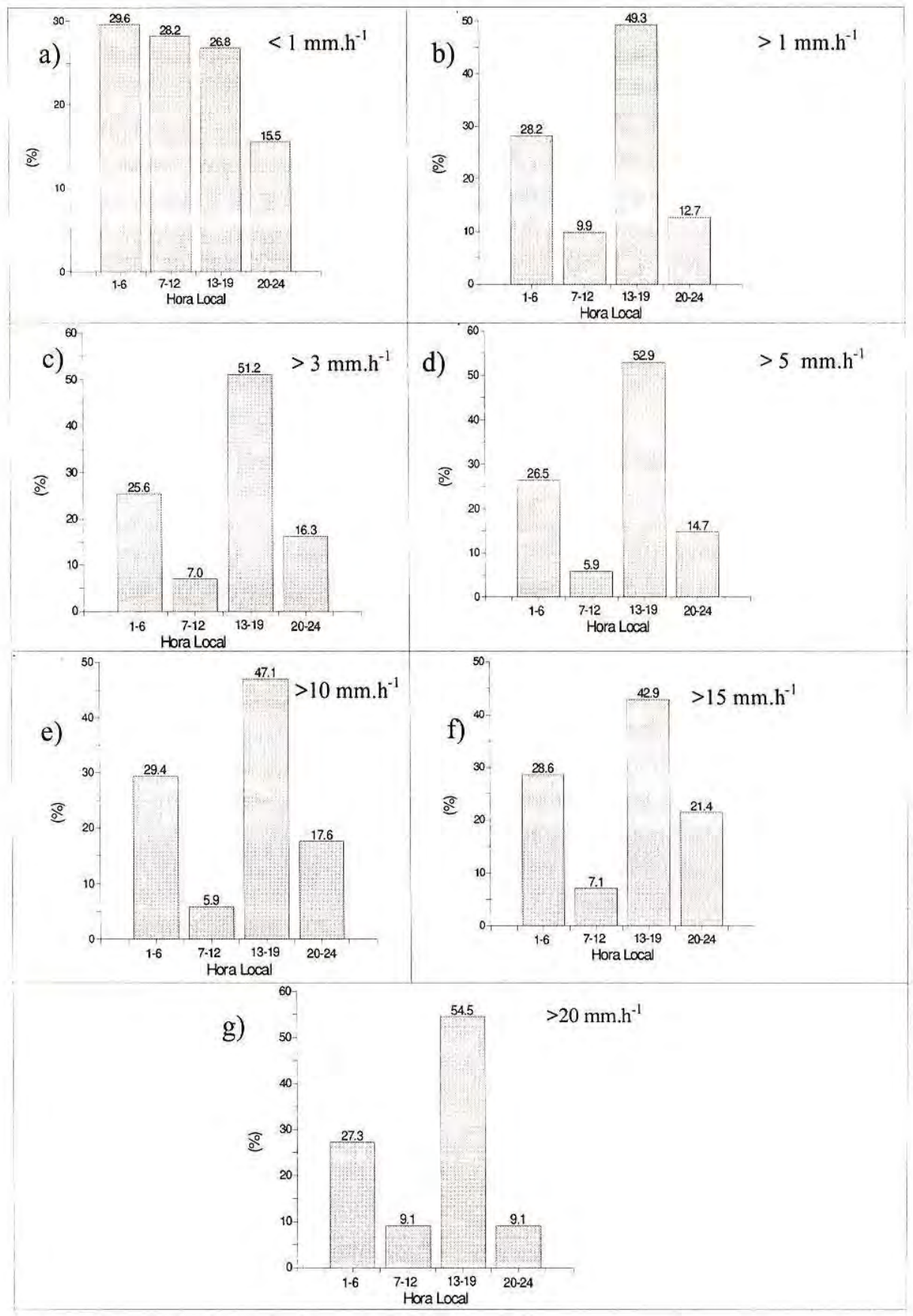

Figura 7 - Frequência Relativa Integrada de Precipitação para os eventos: a) $\left.<1 \mathrm{~mm} \cdot \mathrm{h}^{-1}, \mathrm{~b}\right)>1$, c) $>3$, d) $>5$, e) $>10$, f) $>15$ e g) $>20 \mathrm{~mm} \cdot \mathrm{h}^{-1}$. 
horas, a saber: 0-6, 7-12, 13-19 e 20$24 \mathrm{HL}$. Nos casos inferiores a $1 \mathrm{~mm} /$ $\mathrm{h}$ de precipitação, há uma distribuição mais regular e com a menor ocorrência durante à noite. Os eventos com precipitação horária acima de 1,3 e 5 $\mathrm{mm} \cdot \mathrm{h}^{-1}$ mostram uma predominância nos intervalos 1-6 e 13-19. Os eventos com precipitação acima de 10,15 e 20 $\mathrm{mm} \cdot \mathrm{h}^{-1}$, praticamente, mostram um mesmo padrão entre si, com os maiores números de casos durante 16 e $13-18$ HL.

\section{CONCLUSÕES}

Este estudo analisa a ocorrência de chuva durante o experimento TRMM/ LBA, realizado em área de pastagem na região de Ji-Paraná (RO) durante os meses de janeiro e fevereiro de 1999 (estação chuvosa). Os resultados mostraram que a precipitação ocorrida foi acima da média climatológica, sendo que a origem da formação dessas chuvas foi predominantemente convectiva (representa cerca de $66 \%$ do volume total de chuva) com máximo de freqüência de ocorrência às $15 \mathrm{HL}$. Ocorreu um curto periodo de estiagem (8 a 14/02), seguido da passagem de 2 sistemas de mesoescala (15 e 18/02). Estes eventos de mesoescala provocaram chuvas fortes durante a madrugada (máximo às $4 \mathrm{HL}$ ) e representaram $33 \%$ do volume total de chuva. Estes resultados sugerem que a atmosfera, sobre a área em estudo, organizou-se de 2 maneiras na produção de chuva: através da convecção isolada, com um máximo de precipitação à tarde (16 HL); e através da organizaçao da convecção por Sistemas Convectivos de Mesoescala (SCM), os quais provocaram chuvas estratiformes fracas e também eventos fortes no periodo noturno (4 HL). Além disso, estes resultados concordam com observações independentes de precipitação realizadas recentemente por Rickenbach et al. (2000), através de radar meteorológico sobre a mesma área de estudo deste trabalho, os quais sugerem que estes SCM's posssuíam características estratiformes e chuvas mais fraca. Este mesmo trabalho lança a hipótese de que o escoamento atmosférico de larga escala possam estar modulando o tipo de convecção (isolada e profunda local ou do tipo sistemas convectivos de mesoescala) predominante durante $o$ TRMM-LBA.

\section{AGRADECIMENTOS}

Os autores desejam agradecer à todos aqueles que ajudaram na implementação do experimento TRMM/LBA. Os autores agradecem aos "referees" por seus comentários e sugestões. Julio Tóta (bolsa de doutorado 142398/98-5) e Gilberto Fisch (bolsa 300564/96-1) agradecem ao $\mathrm{CNPq}$ por apoiar esta pesquisa. Agradecimento especial à FAPESP pelo projeto Interação BiosferaAtmosfera de Mesoescala na Amazônia (97/9926-9). 


\section{Bibliografia citada}

Dias de Paiva, E.M.C. 1996. Regime de precipitação na Amazônia e sua relação com o desmatamento e temperatura da superficie do mar: Tese de Doutorado, IPH - Universidade Federal do Rio Grande do Sul. Porto Alegre, Abril de 1996. 205 p.

Ferreira da Costa, R.; Feitosa, J.R.; Fisch, G.; Souza, S. S.; Nobre, C.A. 1998. Variabilidade diária da precipitação em regiões de floresta e pastagem na Amazônia. Acta Amazonica 28(4): 395 407.

Fisch, G.; Lean, J.; Wright, I.R.; Nobre, C.A. 1997. Simulaçòes climáticas do efeito do desmatamento na região Amazônica: Estudo de um caso em Rondônia. Revista Brasileira de Meteorologia, 12 (1): 33-48

Fisch, G.; Marengo, J.A.; Nobre, C.A. 1998. Uma revisão geral sobre o clima da Amazônia. Acta Amazonica 28(2):101126

Gash, J.H.C.; Nobre, C.A. 1997. Amazonian Deforestation and Climate. Bulletin of the American Meteorological Society. 75 (5): 823-830

Molion, L.C.B. 1987. Micrometeorologia of a Amazonian rain forest. In: Dickinson, R.E. (ed.). The Geophysiology of Amazonia: Vegetation and Climate Interactions. New York, Willey \& Sons, 255272

Nobre, C.A.; Sellers, P.J.; Shukla, J. 1991. Amazonian deforestation and regional climate change. Journal of Climate, 4 (10): 957-988

Rickenbach, T. M.; Cifelli, R. C.; Halverson, J. B.; Kucera, P. A.; Thieli, O. W. 2000. Convective systems in contrasting regimes during TRMM-LBA in Rondônia, Brasil. $15^{T H}$ Conference on Hydrology, AMS 80 TH Annual Meeting, Long Beach - USA. p. $329-332$
Tóta, J. 1998. Estimativa regional de fluxos de calor sensivel e latente em áreas de floresta e pastagem na Amazónia. (Dissertação de Mestrado em Meteorologia) - Instituto Nacional de Pesquisas Espaciais, Sào José dos Campos-SP.. 113p. 